\title{
Shaowu as a Sinitic language
}

In this book, we will refer to the language spoken in the city of Shaowu and its environs as 'Shaowu', just like we usually call, in English, the official language of the People's Republic of China as 'Mandarin' or 'Mandarin Chinese', and not the Mandarin (Chinese) language. The Ethnologue has established a measure of inherent intelligibility with other varieties of less than $85 \%$ as likely to signal difficulty in comprehension of the indicated language. ${ }^{2}$ The mutual intelligibility between Shaowu and other Sinitic languages, such as Cantonese, Fuqing Min and Mandarin (all of which the author of this monograph speaks at a native level) is estimated to be lower than such a figure. On her first visit to Shaowu city in 2009, the author only understood about $30 \%$ of the conversations and was only able to comprehend and speak it with fluency after four years of intermittent fieldwork. The author is not alone in her case: many immigrants to Shaowu from other parts of China have not got to understand or speak Shaowu after many years of residence in the city. If the inherent intelligibility is relatively low for speakers from other Sinitic groups and if it takes a fair amount of time to reach an acquired intelligibility and proficiency, it is accurate and appropriate, linguistically speaking, to refer to Shaowu as a language on its own within the Sinitic family.

According to the Language Atlas of China (2012: 3), the Sinitic family comprises ten linguistic groups, arranged in descending order of number of speakers:

\begin{tabular}{lc}
\hline Sinitic language group & Number of speakers (in millions) \\
\hline Mandarin, a.k.a. Guanhua & 798.59 \\
Min & 75.0 \\
Wu & 73.79 \\
lin & 63.05 \\
Yue & 58.82 \\
Gan & 48.0 \\
Hakka & 42.2 \\
Xiang & 36.37 \\
Pinghua \& Tuhua & 7.78 \\
Hui & 3.3 \\
\hline
\end{tabular}

2 https://www.ethnologue.com/about/language-info\#Dialects (Last access on 8 June 2020). 
